\title{
Effects of angular vibration on the flow, segregation, and interface morphology in vertical Bridgman crystal growth
}

\author{
W.C. $\mathrm{Yu}^{\mathrm{a}}{ }^{\text {, Z.B. Chen }}{ }^{\mathrm{a}}$, W.T. Hsu ${ }^{\mathrm{b}}$, B. Roux ${ }^{\mathrm{c}}$, T.P. Lyubimova ${ }^{\mathrm{d}}$, C.W. Lan ${ }^{\mathrm{b}, *}$ \\ ${ }^{a}$ Department of Molecular Science and Engineering, National Taipei University of Technology, Taiwan, ROC \\ ${ }^{\mathrm{b}}$ Department of Chemical Engineering, National Taiwan University, Taipei 10617, Taiwan, ROC \\ ${ }^{\mathrm{c}}$ Laboratoire Modélisation et simulation numérique en mécanique, L3M: CNRS-Universités d'Aix-Marseille, France \\ ${ }^{\mathrm{d}}$ Institute of Continuous Media Mechanics UB RAS, Perm, Russia
}

Received 18 May 2006

Available online 25 September 2006

\begin{abstract}
The effect of angular vibration on the flow, segregation, and interface morphology during vertical Bridgman crystal growth was investigated. Transparent experiments using SCN containing about $0.02 \mathrm{wt} . \%$ acetone were performed. To simulate the observed results, both direct numerical simulation (DNS) and Schlichting boundary layer approximation (SBLA) were considered in the computer model. The simulated morphological breakdown patterns, as a result of acetone accumulation (segregation), are consistent with the experimental observation. At high frequency with low amplitudes, both simulation approaches gave consistent results. However, care must be taken in using SBLA for low frequency vibration of several hertz.
\end{abstract}

(C) 2006 Elsevier Ltd. All rights reserved.

Keywords: Angular vibration; Convection; Morphology; Segregation; Directional solidification

\section{Introduction}

The vertical Bridgman or gradient freeze technique is a popular method for crystal growth. The simplicity in operation and the low thermal gradients are particular suitable to the growth of compound semiconductor crystals [1,2]. The stabilized thermal configuration is also a great advantage offering weak convection and less growth striations due to flow stability. However, the lack of melt stirring could often cause large radial and axial non-uniformities due to the segregation of dopants. For the heavy-doping situation, the local accumulation of dopants can also accelerate constitutional supercooling, which leads to the morphological breakdown of the planar interface [3-6]. Therefore, an active control over the melt convection is extremely important for this growth process. Several

\footnotetext{
${ }^{*}$ Corresponding author. Tel./fax: +88622363 3917.

E-mail address: cwlan@ntu.edu.tw (C.W. Lan).
}

control methods have been proposed for vertical Bridgman crystal growth, including using magnetic fields e.g., [7,8], centrifugal forces [9-11], and the accelerated crucible rotation technique (ACRT) [12].

Recently, a technique using angular vibration has been proposed and found effective in reversing the flow and segregation near the growth interface $[13,14]$. However, the detailed numerical analysis and parameter studies have not yet been carried out. In principle, the angular vibration technique (AVT) is very similar to ACRT, but ACRT uses a much lower frequency. In ACRT, in order to develop the Eckman boundary layer, the time for a spin-up or spindown cycle is usually in the order of $2 R_{\mathrm{c}} / \sqrt{\Omega_{\mathrm{R}} v_{\mathrm{m}}}$, where $R_{\mathrm{c}}$ is the crystal radius, $\Omega_{\mathrm{R}}$ the rotation speed, and $v_{\mathrm{m}}$ the kinematic viscosity of the melt. This time constant is usually several tens of seconds for most of the materials having the diameter of several centimeters. On the other hand, based on the same operation, AVT uses a much higher frequency. Such a vibration causes a streaming flow at the growth interface, and thus effective in the local flow and 


\begin{tabular}{|c|c|c|c|}
\hline \multicolumn{4}{|c|}{ Nomenclature } \\
\hline$b_{\mathrm{v}}$ & vibration amplitude & $v_{\theta}$ & azimuthal velocity \\
\hline$C$ & solute concentration & $z$ & cylindrical coordinate \\
\hline$C_{p}$ & specific heat & & \\
\hline$D^{r}$ & solute diffusivity in the melt & \multicolumn{2}{|c|}{ Greek symbols } \\
\hline $\boldsymbol{e}_{z}$ & unit vector in $z$-direction & $\alpha$ & thermal diffusivity \\
\hline$f$ & vibration frequency & $\beta_{\mathrm{T}}$ & thermal expansion coefficient \\
\hline$F$ & unknown function & $\beta_{\mathrm{S}}$ & solutal expansion coefficient \\
\hline$g_{0}$ & gravitational acceleration & $\delta$ & Schlichting boundary layer thickness \\
\hline$\Delta H$ & heat of fusion & $\kappa$ & normalized crystal thermal conductivity, $k / k_{\mathrm{m}}$ \\
\hline$h_{\mathrm{c}}$ & height of growth front & $\varphi$ & angle between the tangent to the growth front \\
\hline$k$ & thermal conductivity & & and the axis of rotation \\
\hline$K$ & equilibrium segregation coefficient & $v$ & kinematic viscosity, $\mu_{\mathrm{m}} / \rho_{\mathrm{m}}$ \\
\hline$m$ & $\begin{array}{l}\text { slope of the liquidus line in the phase dia- } \\
\text { oram }\end{array}$ & $\mu$ & $\begin{array}{l}\text { viscosity } \\
\text { density }\end{array}$ \\
\hline$n$ & unit normal vector & $\Psi$ & stream function \\
\hline $\operatorname{Pr}$ & Prandtl number, $v_{\mathrm{m}} / \alpha_{\mathrm{m}}$ & $\omega$ & vorticity \\
\hline$r$ & cylindrical coordinate & $\Omega$ & angular vibration frequency \\
\hline $\begin{array}{l}R a_{\mathrm{T}} \\
R a_{\mathrm{S}}\end{array}$ & $\begin{array}{l}\text { thermal Rayleigh number, } \beta_{\mathrm{T}} R_{\mathrm{c}}^{3} g_{0} T_{\mathrm{m}} / v_{\mathrm{m}} \alpha_{\mathrm{m}} \\
\text { solutal Rayleigh number, } \beta_{\mathrm{s}} R_{\mathrm{c}}^{3} g_{0} C_{0} / v_{\mathrm{m}} \alpha_{\mathrm{m}}\end{array}$ & $\Omega_{\mathrm{R}}$ & rotation speed \\
\hline$R_{\mathrm{c}}$ & radius of crystal & \multicolumn{2}{|c|}{ Superscript } \\
\hline$t$ & time & $*$ & dimensionless variables \\
\hline$S c$ & Schmidt number, $v_{\mathrm{m}} / D$ & & \\
\hline$S t$ & Stefan number, $\Delta H /\left(C_{p_{\mathrm{m}}} T_{\mathrm{m}}\right)$ & \multicolumn{2}{|c|}{ Subscripts } \\
\hline$T$ & temperature & 0 & initial value \\
\hline$T_{\text {eff }}$ & effective heater temperature & amp & ampoule \\
\hline$T_{\mathrm{m}}$ & melting point & $\mathrm{c}$ & crystal \\
\hline$U$ & translation speed & $\mathrm{h}$ & heater \\
\hline$u$ & $r$-component of velocity & $\mathrm{m}$ & melt \\
\hline$u_{\mathrm{t}}$ & tangential velocity at the Schlichting boundary & $\max$ & maximum \\
\hline & layer & $\min$ & minimum \\
\hline$v$ & $z$-component of velocity & $\infty$ & away from the boundary \\
\hline
\end{tabular}

segregation control. Because the bulk flow is only slightly affected, the global segregation is not enhanced.

In this paper, we took a transparent growth system, consisting of acetone-doped succinonitrile, as an example to investigate the effects of vibration frequencies on the flow and segregation control. In addition to experimental observations, some numerical simulations are reported. Both direct numerical simulation (DNS) and the Schlichting boundary layer approximation (SBLA) were carried out and compared. The calculated amplitudes of the oscillatory flow intensity and the interface, as well as the solute segregations, should be informative to crystal growers who are interested in using this technique. In the next section, the governing equations and boundary conditions for the vertical Bridgman growth are introduced. The equivalent boundary condition for SBLA is derived and the numerical method is described briefly. Section 3 gives a brief description of the experimental setup and solidification experiments. The results and discussion are arranged in Section 4 before a short conclusion.

\section{Governing equations and boundary layer approximation}

The schematic of the vertical Bridgman crystal growth used in this study is depicted in Fig. 1. The furnace environment used for simulation was described by an effective heating profile $T_{\mathrm{eff}}(z, t)$ specified in the model. To start crystal growth from a stationary state (without ampoule translation), this profile was moved upward at speed $U_{\mathrm{h}}$. In the experiments, the furnace was kept stationary, while the ampoule was moving downward at a given speed after the growth began. The angular vibration was applied in the azimuthal angle direction with an amplitude $b_{\mathrm{v}}$ (a fraction of $2 \pi$ ) and angular frequency $\Omega$ (or frequency $f$ in Hertz); $\Omega=2 \pi f$. The system was assumed axisymmetric, so were the flow and temperature fields, as well as the growth front (the melt/crystal interface, $h_{\mathrm{c}}(r, t)$ ). The melt was further assumed incompressible and Newtonian, while the flow was laminar. The Boussinesq approximation was also adopted. Dimensionless variables are defined by scaling length with the crystal radius $R_{\mathrm{c}}$, time $t$ with $R_{\mathrm{c}}^{2} / \alpha_{\mathrm{m}}$, 


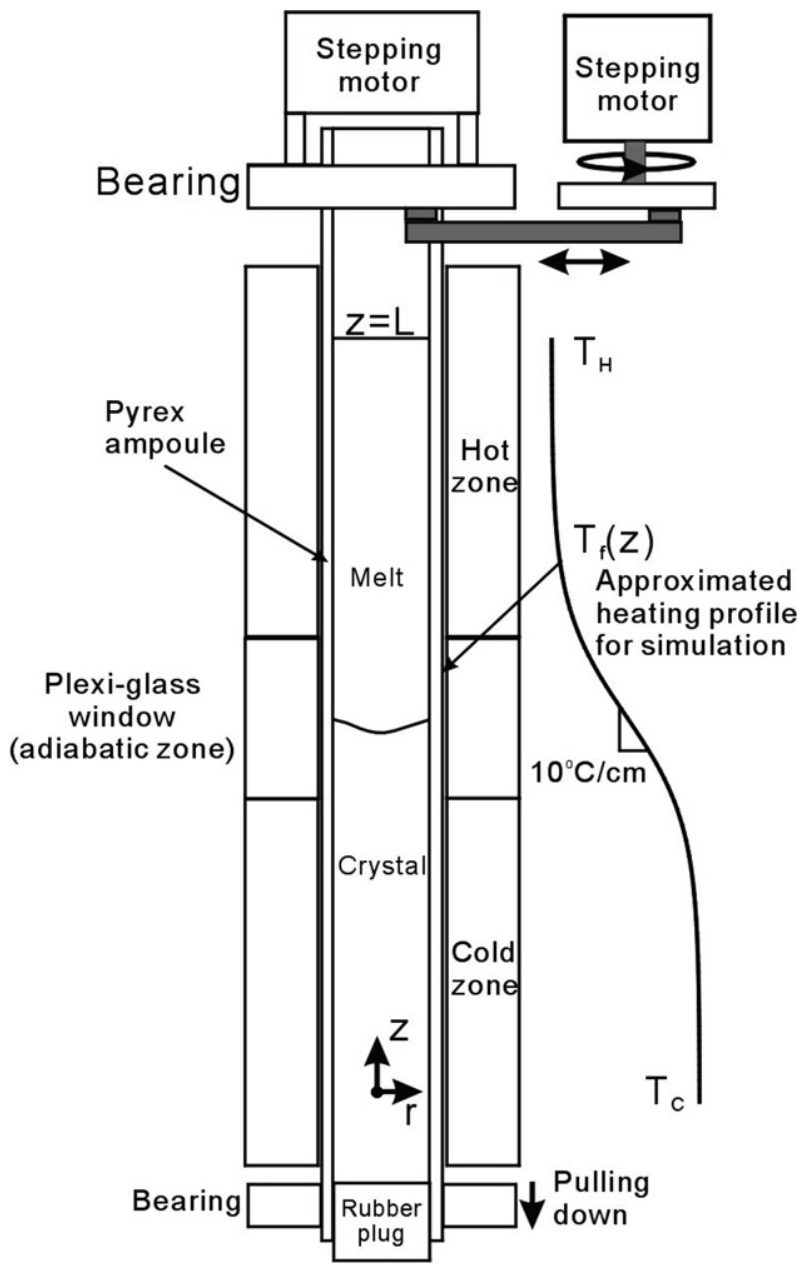

Fig. 1. Sketch of the experimental setup for vertical Bridgman crystal growth with angular vibration; the furnace profile $T_{\mathrm{f}}(z)$ used for simulation is also shown.

velocity with $\alpha_{\mathrm{m}} / R_{\mathrm{c}}$, temperature with the melting point $T_{\mathrm{m}}$, and dopant or solute concentration by the initial concentration $C_{0}$, where $\alpha_{\mathrm{m}}$ is the thermal diffusivity of the melt. For the convenience of representation, all the variables defined afterwards with a superscript $*$ are dimensionless unless otherwise stated. The governing equations for the time-dependent fluid flow and heat and mass transfer in terms of dimensionless stream function $\psi^{*}$, vorticity $\omega^{*}$, azimuthal velocity $v_{\theta}^{*}$, temperature $T^{*}$ and dopant concentration $C^{*}$ can be written as follows:

Equation of motion

$$
\begin{aligned}
& \frac{\partial \omega^{*}}{\partial t^{*}}+\frac{\partial}{\partial r^{*}}\left(\frac{\omega^{*}}{r^{*}} \frac{\partial \psi^{*}}{\partial z^{*}}\right)-\frac{\partial}{\partial z^{*}}\left(\frac{\omega^{*}}{r^{*}} \frac{\partial \psi^{*}}{\partial r^{*}}\right) \\
& \quad+\operatorname{Pr}\left[\frac{\partial}{\partial r^{*}}\left(\frac{1}{r^{*}} \frac{\partial}{\partial r^{*}}\left(r^{*} \omega^{*}\right)\right)+\frac{\partial}{\partial z^{*}}\left(\frac{1}{r^{*}} \frac{\partial}{\partial z^{*}}\left(r^{*} \omega^{*}\right)\right)\right] \\
& \quad+\frac{\partial}{\partial z}\left(\frac{v_{\theta}^{2}}{r}\right)-\operatorname{Pr} R a_{\mathrm{T}} \frac{\partial T^{*}}{\partial r^{*}}+\operatorname{Pr} R a_{\mathrm{S}} \frac{\partial C^{*}}{\partial r^{*}}=0 .
\end{aligned}
$$

Stream equation

$\frac{\partial}{\partial z^{*}}\left(\frac{1}{r^{*}} \frac{\partial \psi^{*}}{\partial z^{*}}\right)+\frac{\partial}{\partial r^{*}}\left(\frac{1}{r^{*}} \frac{\partial \psi^{*}}{\partial r^{*}}\right)+\omega^{*}=0$.
Circulation equation

$$
\begin{aligned}
- & \frac{\partial v_{\theta}^{*}}{\partial t^{*}}+\frac{1}{r^{* 2}}\left[\frac{\partial}{\partial r^{*}}\left(r^{*} v_{\theta}^{*} \frac{\partial \psi^{*}}{\partial z^{*}}\right)-\frac{\partial}{\partial z^{*}}\left(r^{*} v_{\theta}^{*} \frac{\partial \psi^{*}}{\partial r^{*}}\right)\right] \\
& +\operatorname{Pr}\left[\frac{\partial}{\partial r^{*}}\left(\frac{1}{r^{*}} \frac{\partial\left(r^{*} v_{\theta}^{*}\right)}{\partial r^{*}}\right)+\frac{\partial}{\partial z^{*}}\left(\frac{1}{r^{*}} \frac{\partial\left(r^{*} v_{\theta}^{*}\right)}{\partial z^{*}}\right)\right]=0 .
\end{aligned}
$$

Energy equation

$$
\begin{gathered}
-r^{*} \frac{\partial T^{*}}{\partial t^{*}}-\frac{\partial}{\partial r^{*}}\left(r^{*} u^{*} T^{*}\right)-\frac{\partial}{\partial z^{*}}\left(r^{*} v^{*} T^{*}\right)+\frac{\partial}{\partial z^{*}}\left(r^{*} \alpha_{i}\left(T^{*}\right) \frac{\partial T^{*}}{\partial z^{*}}\right) \\
+\frac{\partial}{\partial r^{*}}\left(r^{*} \alpha_{i}\left(T^{*}\right) \frac{\partial T^{*}}{\partial r^{*}}\right)=0, \quad i=(m, c, \mathrm{amp}) .
\end{gathered}
$$

Solute equation

$$
\begin{aligned}
& -r^{*} \frac{\partial C^{*}}{\partial t^{*}}-\frac{\partial}{\partial r^{*}}\left(r^{*} u^{*} C^{*}\right)-\frac{\partial}{\partial z^{*}}\left(r^{*} v^{*} C^{*}\right) \\
& +\frac{P r}{S c}\left[\frac{\partial}{\partial z^{*}}\left(r^{*} \frac{\partial C^{*}}{\partial z^{*}}\right)+\frac{\partial}{\partial r^{*}}\left(r^{*} \frac{\partial C^{*}}{\partial r^{*}}\right)\right]=0 .
\end{aligned}
$$

The solute diffusion in the solid phase was neglected. In the above equations, $\operatorname{Pr}$ is the Prandtl number $\left(\operatorname{Pr} \equiv v_{\mathrm{m}} / \alpha_{\mathrm{m}}\right)$, where $v_{\mathrm{m}}$ is the kinematic melt viscosity, $S c$ the Schmidt number $\left(S c \equiv v_{\mathrm{m}} / D\right)$, and $D$ the solute diffusivity in the melt. Also, $\alpha_{i}$ is the thermal diffusivity of phase $i ; i=c$ for the crystal and $m$ for the melt. Two important dimensionless variables, $R a_{\mathrm{T}}$ and $R a_{\mathrm{S}}$, in the source term of the equation of motion are defined as follows:

$R a_{\mathrm{T}} \equiv \frac{g_{0} \beta_{\mathrm{T}} T_{\mathrm{m}} R_{\mathrm{c}}^{3}}{\alpha_{\mathrm{m}} v_{\mathrm{m}}} ; \quad R a_{\mathrm{S}} \equiv \frac{g_{0} \beta_{\mathrm{S}} C_{0} R_{\mathrm{c}}^{3}}{\alpha_{\mathrm{m}} v_{\mathrm{m}}}$,

where $g_{0}$ is the gravitational acceleration, and $\beta_{\mathrm{T}}$ and $\beta_{\mathrm{S}}$ are the thermal and solutal expansion coefficients, respectively. The stream function $\psi^{*}$ and vorticity $\omega^{*}$ in the above equations are defined in terms of the radial $\left(u^{*}\right)$ and axial $\left(v^{*}\right)$ velocities as:

$u^{*}=-\frac{1}{r^{*}} \frac{\partial \psi^{*}}{\partial z^{*}}, \quad v^{*}=\frac{1}{r^{*}} \frac{\partial \psi^{*}}{\partial r^{*}}$,

$\omega^{*}=\frac{\partial u^{*}}{\partial z^{*}}-\frac{\partial v^{*}}{\partial r^{*}}$.

To solve the above governing equations, boundary conditions are also required. Most of the boundary conditions for melt flow and heat and mass transfer can be found elsewhere [15]. In short, the energy and solute conservation are applied to the growth interface. For example, at the melt/ crystal interface, the interface energy and solute balances can be written as the following, respectively,

$$
\begin{gathered}
\left.\boldsymbol{n} \cdot \nabla T^{*}\right|_{\mathrm{m}}-\left.n \cdot \kappa_{\mathrm{c}} \nabla T^{*}\right|_{\mathrm{c}}+\left.\gamma S t \frac{\partial h_{\mathrm{c}}^{*}}{\partial t^{*}}\left(\boldsymbol{n} \cdot \boldsymbol{e}_{z}\right)\right|_{\mathrm{c}}=0, \\
-\left.\boldsymbol{n} \cdot \nabla C^{*}\right|_{\mathrm{c}}-\left.(1-K) \frac{S c}{P r} C^{*} \frac{\partial h_{\mathrm{c}}^{*}}{\partial t^{*}}\left(\boldsymbol{n} \cdot \boldsymbol{e}_{z}\right)\right|_{\mathrm{c}}=0,
\end{gathered}
$$

where $\boldsymbol{n}$ is the surface normal at the growth front pointing to the melt, $\kappa_{\mathrm{c}} \equiv k_{\mathrm{c}} / k_{\mathrm{m}}$ the normalized crystal thermal conductivity, $\gamma \equiv \rho_{\mathrm{c}} / \rho_{\mathrm{m}}$ the density ratio, and $h_{\mathrm{c}}^{*}$ the dimensionless interface height. The Stefan number $S t \equiv \Delta H /$ $\left(C_{p_{\mathrm{m}}} T_{\mathrm{m}}\right)$ scales the heat of fusion $(\Delta H)$ released during 
solidification to the sensible heat in the melt. The equilibrium segregation coefficient $K \equiv C_{\mathrm{c}} / C$ was obtained according to the phase diagram; $K=0.1$ for acetone in $\mathrm{SCN}$. At the ampoule/material interfaces, heat flux continuity is forced, while the solute flux was set to zero. The melt surface was set to stress free. The no-slip boundary condition is used for the solid boundaries of the interface. Therefore, the angular vibration of the crucible can be described by the sinusoidal azimuthal velocity as follows:

$v_{\theta}^{*}=2 \pi b_{\mathrm{v}} f^{*} r^{*} \sin \left(2 \pi f^{*} t^{*}\right)$,

where $2 \pi b_{\mathrm{v}}$ is the angular vibration amplitude and $f^{*}$ the dimensionless vibration frequency scaled by $\alpha_{\mathrm{m}} / R_{\mathrm{c}}^{2}$. In fact, in term of the accelerated ampoule rotation, $2 \pi b_{\mathrm{v}} f^{*}$ corresponds to the maximum rotation speed (dimensionless). In other words, beside the much smaller period for reversing rotation in AVT and ACRT are quite similar. The detailed comparisons of the calculated flow and solute fields in the growth of $\mathrm{ZnCdTe}$ using both techniques have been discussed in our previous report [14]. Because the solidification time in experiments lasted for hours, it was too time consuming to perform direct numerical simulation (DNS) using Eq. (10) as the boundary condition because the time step used for time integration needs to be much smaller than the vibration period. Instead, if the frequency is high enough such that the thickness of viscous boundary layer is small in comparison with all characteristics, an averaged flow approach using the Schlichting boundary layer approximation is possible.

Based on Schlichting formula [16], we can search for a pure azimuthal solution for the pulsational velocity having the following form:

$v_{\theta}^{*}=2 \pi b_{\mathrm{v}} f^{*} \operatorname{Re}\left\{F^{*}\left(z^{*}, r^{*}\right) \mathrm{e}^{\mathrm{i} 2 \pi f^{*} t^{*}}\right\}$.

The unknown function $F^{*}$ in Eq. (11) can be obtained by plugging the above equation into the linearized NavierStokes equation, i.e.,

$\mathrm{i} 2 \pi f^{*} F^{*}=\operatorname{Pr}\left(\frac{\partial^{2} F^{*}}{\partial r^{* 2}}+\frac{1}{r^{*}} \frac{\partial F^{*}}{\partial r^{*}}+\frac{\partial^{2} F^{*}}{\partial z^{* 2}}-\frac{F^{*}}{r^{* 2}}\right)$.

The boundary condition at the growth front is $F^{*}\left(0, r^{*}\right)=r^{*}$, and this allows us to find the linear solution satisfying

$0=\operatorname{Pr}\left(\frac{\partial^{2} F^{*}}{\partial r^{* 2}}+\frac{1}{r^{*}} \frac{\partial F^{*}}{\partial r^{*}}-\frac{F^{*}}{r^{* 2}}\right)$

and

$\mathrm{i} 2 \pi f^{*} F^{*}=\operatorname{Pr}\left(\frac{\partial^{2} F^{*}}{\partial z^{* 2}}\right)$.

The solution of the above equations gives $F^{*}=r^{*} \mathrm{e}^{-(1+\imath) z^{*} \delta^{*}}$, where $\delta^{*}$ is the dimensionless boundary layer thickness, i.e., $\delta^{*}=\sqrt{\frac{P r}{\pi f^{*}}}$. The dimensional form of the Schlichting boundary layer gives $\delta=\sqrt{\frac{v_{\mathrm{m}}}{\pi f}}$. Therefore, the linear angular velocity is obtained. With the pulsational angular velocity, the time average effect $\left(\overline{v_{\theta}^{* 2} / r^{*}}\right)$ on the radial component of the Navier Stokes equation can be easily obtained, i.e., $\overline{v_{\theta}^{* 2} / r^{*}}=0.5 b_{\mathrm{v}}^{2}\left(2 \pi f^{*}\right)^{2} r^{*} \mathrm{e}^{-2 z^{*} / \delta^{*}}$. Then, if one uses this as the centrifugal force term for the radial component of the Navier-Stokes equation, the radial velocity in the Schlichting layer is obtained:

$u^{*}=\frac{b_{\mathrm{v}}^{2}\left(\pi f^{*}\right) r^{*}}{2}\left(1-\mathrm{e}^{-2 z^{*} / \delta^{*}}\right)$.

Away from the boundary layer, the radial velocity is given by $u_{\infty}^{*}=\frac{b_{v}^{2}\left(\pi f^{*} r^{*}\right.}{2}$. When the boundary is curved, the angle $\varphi$ between the tangent to the growth front and the axis of rotation needs to be considered. With the above approximation, if the Schlichting boundary layer is very thin, we can take this slip tangential velocity $\left(u_{\mathrm{t}}^{*}\right)$ for the growth front as follows:

$u_{\mathrm{t}}^{*}=\frac{b_{\mathrm{v}}^{2}\left(\pi f^{*}\right) r^{*}}{2} \sin (\varphi)$.

With this boundary condition, the simulation cost of the angular vibration is greatly reduced because the time step is no longer restricted by the vibration period; the simulation time can be reduced from about a week to several hours. We refer to this approach as the Schlichting boundary layer approximation (SBLA). However, there are some limitations in this approximation. When the frequency is not high enough, the boundary layer becomes too thick, so that the use of this boundary condition can be erroneous. We will examine this approximation later by comparing the result using this boundary condition with the one from the direct numerical simulation using Eq. (10) as the boundary condition. Furthermore, since the linearized Navier-Stoke equation is used, the vibration amplitude needs to be small as well.

The above governing equations and boundary conditions are discretized by a finite volume method, and the resultant differential/algebraic equations are solved by DASPK solver with adaptive stepsize control [15]. The total number of unknowns after the finite volume approximation is 34052, and all the calculations are performed using a personal computer ( $\mathrm{P} 4-3 \mathrm{GHz} \mathrm{CPU})$.

\section{Experimental}

Succinonitrile (SCN) containing about $0.02 \mathrm{wt} . \%$ acetone was directionally solidified in a transparent vertical Bridgman system, as sketched in Fig. 1. Before experiments, SCN (Furuka Inc., about 99\% purity) was purified first by vacuum distillation at $50 \mathrm{mTorr}$ for ten times. The sample purity was checked by its melting point using a thermistor showing the sample purity may be higher than $99.99 \%$ [17]. The distilled sample was collected in a $17-\mathrm{mm}$ diameter Pyrex ampoule $(2.5 \mathrm{~mm}$ in thickness) and further purified by four-zone refining for more than sixty passes. The sample was then examined by directional solidification. No morphological breakdown was observed up to a solidification speed of $7 \mu \mathrm{m} / \mathrm{s}$ for a thermal gradient of 8 to $10 \mathrm{~K} / \mathrm{cm}$. To perform the solidification experiments, 
about 0.02 wt. $\%$ of acetone was injected into the sample through a $5 \mu 1$ micro-syringe inserted into the bottom of the sample. To ensure the acetone uniformity in the melt, at least one day was waited before the growth experiment. The total sample length was about $20 \mathrm{~cm}$.

The furnace for directional solidification consisted of two heating zones made of cupper blocks each with a Nichrome wire inside as a heating element. In between, a transparent insulation zone made of Plexiglas was used. The hot- and cold-zone temperatures were controlled independently by two PID controllers and the temperatures were set to $80^{\circ} \mathrm{C}$ (top) and $40^{\circ} \mathrm{C}$ (bottom), respectively. The thermal gradient at the interface was measured by an immersed thermocouple traveling with the sample. By taking an average of the gradients at the interface from solidification and melting curves, we obtained a thermal gradient of about $8-10 \mathrm{~K} / \mathrm{cm}$. To translate the ampoule accurately, a micro-stepping motor was used to drive a screw slide; the translation rate was controlled at $2 \mu \mathrm{m} / \mathrm{s}$ in this study. During solidification, a video camera was used to record the evolution of the interface morphology with a back lighting to enhance the contrast of the image. Also, to allow smooth rotation, the ampoule was tightly fitted into a pair of bearings (top and bottom) that were both mounted on the translating system. Furthermore, to generate angular vibration, as shown in Fig. 1, a stepping motor was used to rotate a disk having a shaft connected to another disk where the sample was mounted. The vibration amplitude was controlled by the mounting position of the shaft on the disk, while the frequency was controlled by the rotation speed of the stepping motor. In the experiments, the dimensionless amplitude was about 0.035 .

\section{Results and discussion}

\subsection{Experimental observations}

Fig. 2 shows the effect of angular vibration on the interface evolution. As shown in the top left photograph, without vibration $(0 \mathrm{~Hz})$, the interface was flat. This is due to the closed thermal conductivities of the melt and the crystal. When the ampoule started to translate downward at $2 \mu \mathrm{m} / \mathrm{s}$, the solidification began and the interface became concave due to the release of heat fusion, as shown by the photograph at $50 \mathrm{~min}$ after pulling. An obvious depression or pit formed at the interface center, and this was due to acetone accumulation, which lowered the solidification temperature. As the solidification proceeded further, more solute was accumulated in the front of the interface, because the acetone has a lower solubility in the solid than in the melt (the segregation coefficient is 0.1). Finally, the freezing interface at the tip of the pit broke down. This phenomenum was also observed in our previous report for the case of $0.007 \mathrm{wt} . \%$ acetone [11], but with a slightly higher pulling speed at $2.5 \mu \mathrm{m} / \mathrm{s}$. The time for pit formation and interface breakdown in the present case were much shorter due to the significantly higher acetone concentra-
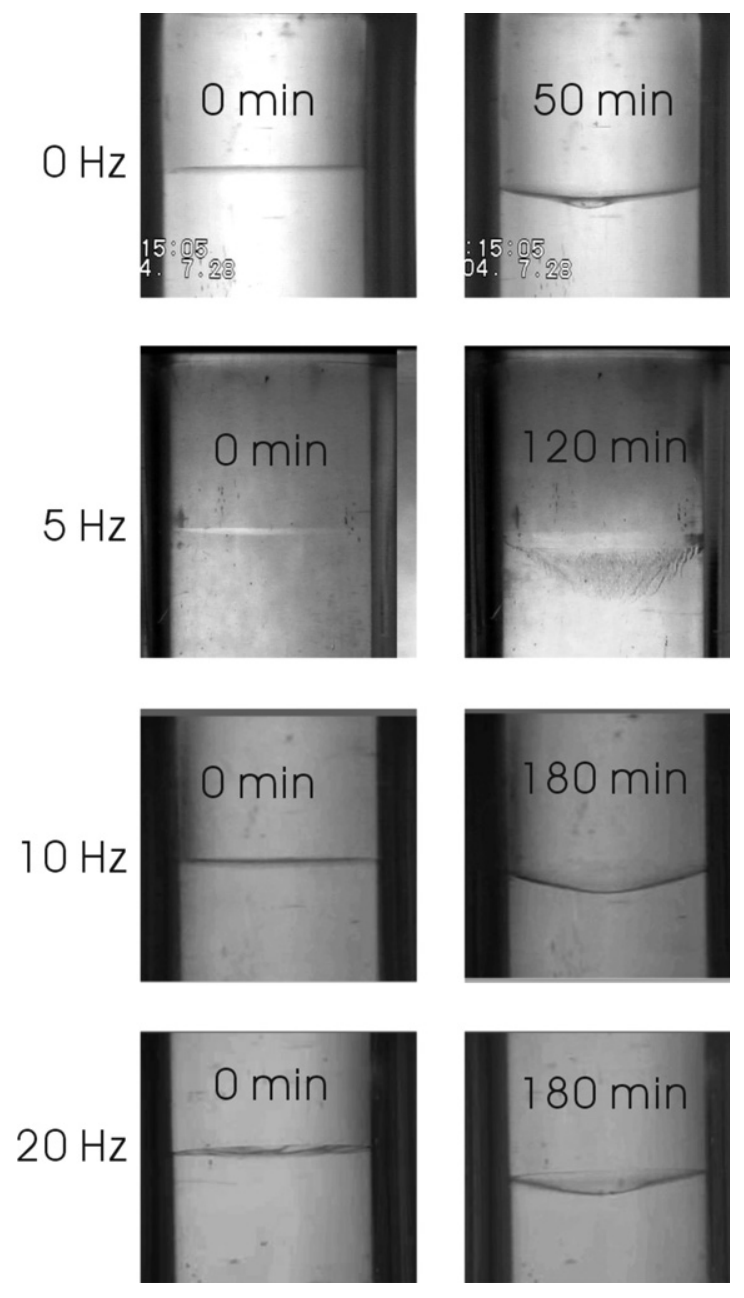

Fig. 2. Effect of angular vibration frequency on the interface morphologies at different times for SCN growth containing $0.02 \mathrm{wt} . \%$ acetone at an ampoule pulling speed of $2.0 \mu \mathrm{m} / \mathrm{s}$.

tion. With angular vibration at $5 \mathrm{~Hz}$ (the dimensionless amplitude of the vibration is about 0.035), the interface remained flat at stationary. However, as the ampoule moved, the interface deformed and started to break down at about $50 \mathrm{~min}$, but the pit was significantly wider and flatter. Such a breakdown continued and eventually led to a dendritic structure, as shown in the photograph marked $120 \mathrm{~min}$. With $10 \mathrm{~Hz}$ vibration, while the vibration amplitude remained the same, the interface shape was about the same at stationary (without ampoule movement), and the interface became concave with translation. However, no pit formation and no breakdown were observed in 3 hours of solidification. Similar results were also observed for $20 \mathrm{~Hz}$ vibration, as shown in the bottom photographs. However, at stationary, the interface became rugged, sometimes wavy, along the angular direction. These interface morphologies at $20 \mathrm{~Hz}$ were consistent with those reported previously using a $60-\mathrm{Hz}$ electromagnetic vibrator [13]. The rugged interface is believed to be caused by the angular hydrodynamic instability; however, its detailed mechanism remains unclear. 


\subsection{Numerical simulation}

The numerical simulation of the experiments was also carried out. For the case of $0 \mathrm{~Hz}$, the predicted pit formation and interface breakdown, using the supercooling as the criterion, agreed reasonably well with the experiments. The corresponding simulation results are shown in Fig. 3a. At stationary, the interface was almost flat, only slightly convex. As a result, a small weak flow cell was induced near the interface. The upper cell shown on the right hand side of the plot at $t=0$ in Fig. 3a was caused by the radial heating. The acetone concentration was uniform as well at stationary. One hour after solidification commenced, the interface became significantly concave, with a pit formed at the center. The lower flow cell induced by the interface concavity caused acetone accumulation at the pit and accelerated supercooling and the morphological breakdown. This is consistent with the experimental observation.

For $5 \mathrm{~Hz}$ vibration, direct numerical simulation (DNS) was also carried out. Although the frequency was not high, the simulation time was extremely long because the solidification time lasted several hours and the time step for integration needed to be much smaller than the vibration period $(0.2 \mathrm{~s})$. More than $10^{5}$ time steps were needed for one simulation. The time averaged flow and solute profiles are shown in Fig. $3 \mathrm{~b}$ at different times. It was observed that the supercooling started at around $2400 \mathrm{~s}$ as shown in Fig. 3b. A small secondary flow cell was observed near the center of the interface, while other flow structures remained almost unchanged. Numerical breakdown also appeared at latter time (e.g., at 4800 s). Pit formation was not obvious, which agreed reasonably well with the experimental observation. The time for the onset of supercooling was also consistent with the observation.

The simulation using the Schlichting boundary layer approximation (SBLA) was also carried out for $5 \mathrm{~Hz}$ vibration. As shown in Fig. 4a, the results are quite different from that in Fig. 3b. In SBLA, the frequency was assumed high enough, so that the tangential velocity was imposed at the growth interface. This resulted in a secondary flow cell sitting upon the interface. No pit formation and supercooling were found in Fig. 4a. In other words, the simulated results using SBLA were not consistent with the experiments. The simulation seemed to over predict the effect of the angular vibration.

For the angular vibration of $20 \mathrm{~Hz}$, as shown in Fig. 4b, the results are similar to that of $5 \mathrm{~Hz}$ in Fig. $4 \mathrm{a}$, but the flow intensity for the Schlichting cell is significantly larger. Again, no pit formation and supercooling were observed, which was consistent with the experiment. The interface shape was also in reasonable agreement with the observed one. Direct numerical simulation of the growth at $20 \mathrm{~Hz}$ was not realistic because of the long computation time. Nevertheless, we still examined the validity of SBLA for $20 \mathrm{~Hz}$ for a similar case. To save computation time, we picked a stationary system similar to the case at $t=0 \mathrm{~s}$ in Fig. 4b for comparison, but the solutal effect was ignored (let $C=0$ ). Fig. 5 shows the comparison of the calculated isotherms (left) and the streamlines (right) using SBLA and DNS at 5 and $20 \mathrm{~Hz}$ for the same vibration intensity a

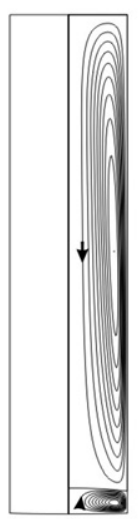

Os
$\mathrm{OHz}$

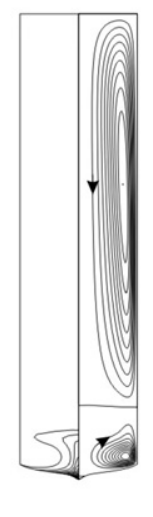

3600 s

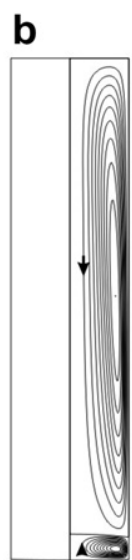

Os

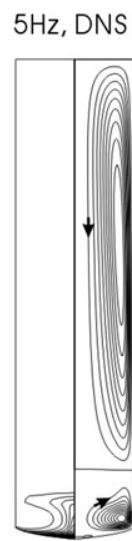

$2400 s$

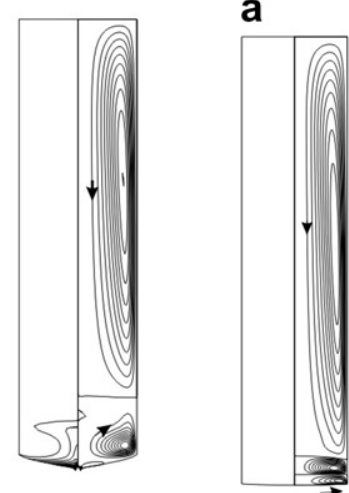

Os

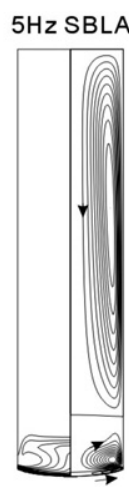

2400s

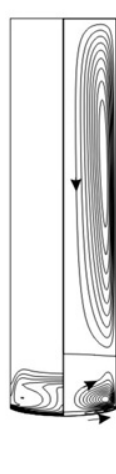

4800s

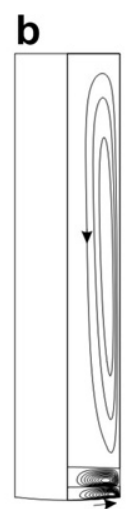

Os

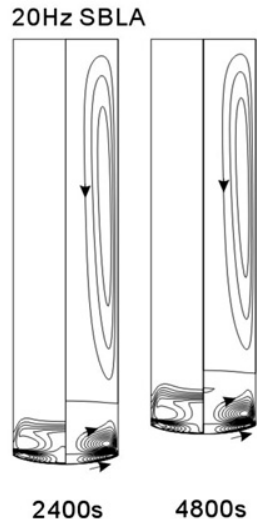

Fig. 3. Calculated flow and acetone fields, as well as the interface shape using direct numerical simulation (DNS) for (a) without angular vibration; (b) $5 \mathrm{~Hz}$ vibration; the ampoule pulling speed is $2.0 \mu \mathrm{m} / \mathrm{s}$ and vibration amplitude is about 0.035 . In (a) at $t=0, \Psi_{\min }=-1.8 \times 10^{-4} \mathrm{~g} / \mathrm{s}$, $\Psi_{\max }=3.037 \times 10^{-5} \mathrm{~g} / \mathrm{s}$, and $C / C_{0}=0$ and at $t=1 \mathrm{~h}, \Psi_{\min }=-1.8 \times$ $10^{-4} \mathrm{~g} / \mathrm{s}, \Psi_{\max }=3.037 \times 10^{-5} \mathrm{~g} / \mathrm{s}$, maximum $C / C_{0}=16.2693$, and minimum $C / C_{0}=1$. In (b) $t=0, \Psi_{\min }=-1.8 \times 10^{-4} \mathrm{~g} / \mathrm{s}, \Psi_{\max }=3.037 \times$ $10^{-5} \mathrm{~g} / \mathrm{s}$, and $C / C_{0}=0 ; \quad$ at $t=2400 \mathrm{~s}, \quad \Psi_{\min }=-1.416 \times 10^{-4} \mathrm{~g} / \mathrm{s}$, $\Psi_{\max }=3.309 \times 10^{-4} \mathrm{~g} / \mathrm{s}$, maximum $C / C_{0}=8.1844$, and minimum $C / C_{0}=1 ; \quad$ at $t=4800 \mathrm{~s}, \quad \Psi_{\min }=-1.411 \times 10^{-4} \mathrm{~g} / \mathrm{s}, \quad \Psi_{\max }=3.291 \times$ $10^{-4} \mathrm{~g} / \mathrm{s}$, maximum $C / C_{0}=18.2487$, and minimum $C / C_{0}=1$.

Fig. 4. Simulation results based on the Schlichting boundary layer approximation: (a) $5 \mathrm{~Hz}$; (b) $20 \mathrm{~Hz}$ angular vibration; The ampoule pulling speed is $2.0 \mu \mathrm{m} / \mathrm{s}$ and vibration amplitude is about 0.035 . In (a) at $t=0, \Psi_{\min }=-1.79 \times 10^{-4} \mathrm{~g} / \mathrm{s}, \Psi_{\max }=4.0946 \times 10^{-5} \mathrm{~g} / \mathrm{s}$, and $C / C_{0}=0$; at $t=2400 \mathrm{~s}, \Psi_{\min }=-4.16 \times 10^{-4} \mathrm{~g} / \mathrm{s}, \Psi_{\max }=3.540 \times 10^{-4} \mathrm{~g} / \mathrm{s}$, maximum $C / C_{0}=3.9732$, and minimum $C / C_{0}=1$; at $t=4800 \mathrm{~s}, \Psi_{\min }=$ $-4.14 \times 10^{-4} \mathrm{~g} / \mathrm{s}, \Psi_{\max }=3.61 \times 10^{-4} \mathrm{~g} / \mathrm{s}$, maximum $C / C_{0}=5.259$, and minimum $C / C_{0}=1$. In (b) at $t=0, \Psi_{\min }=-4.58 \times 10^{-4} \mathrm{~g} / \mathrm{s}, \Psi_{\max }=$ $1.213 \times 10^{-4} \mathrm{~g} / \mathrm{s}$, and $C / C_{0}=0$; at $t=2400 \mathrm{~s}, \Psi_{\min }=-3.694 \times 10^{-4} \mathrm{~g} / \mathrm{s}$, $\Psi_{\max }=3.763 \times 10^{-4} \mathrm{~g} / \mathrm{s}$, maximum $C / C_{0}=2.678$, and minimum $C / C_{0}=$ 1 ; at $t=4800 \mathrm{~s}, \quad \Psi_{\min }=-3.668 \times 10^{-4} \mathrm{~g} / \mathrm{s}, \quad \Psi_{\max }=3.802 \times 10^{-4} \mathrm{~g} / \mathrm{s}$, maximum $C / C_{0}=3.721$, and minimum $C / C_{0}=1$. 


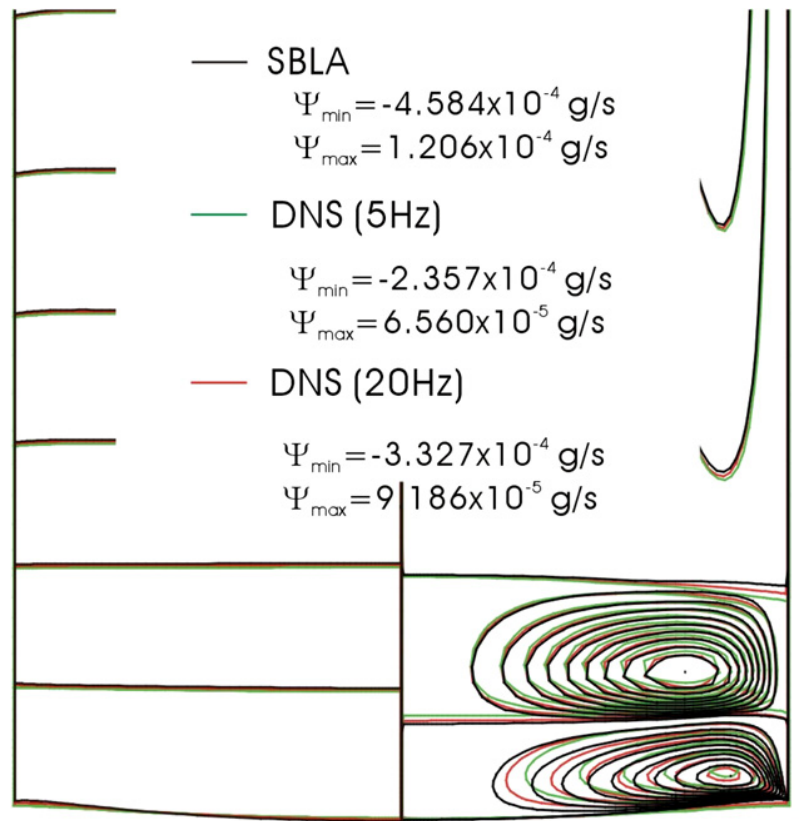

Fig. 5. Comparison of the calculated flow streamlines (right) and isotherms (left) by DNS and SBLA for a stationary condition with the same vibration intensity $\left(2 \pi b_{\mathrm{v}}^{2} f\right) ; b_{\mathrm{v}}=0.035$ and $f=20 \mathrm{~Hz}$ were used for SBLA.

$\left(2 \pi b_{v}^{2} f\right)$; the SBLA is independent of frequency as long as the Schlichting velocity remains the same (also see Eq. (16)). As shown in Fig. 5, the calculated isotherms and flow patterns are all very similar. However, if we compare the flow intensity of the Schlichting cell near the interface, the vortex center moves downward as the frequency is increased from 5 to $20 \mathrm{~Hz}$, which is consistent with the boundary layer thickness predicted in Eq. (15). The flow intensity increases with the frequency as well. However, even at $20 \mathrm{~Hz}$, the maximum flow intensity $\left(\left|\Psi_{\min }\right|\right)$ is only about $72.5 \%$ of that calculated from SBLA. Further increase in the frequency slightly improves the results. The oscillation amplitudes of the stream function for the Schlichting cells are $1.75 \times 10^{-4} \quad\left(\sim 0.74\left|\Psi_{\min }\right|\right)$ and $6.03 \times 10^{-5} \mathrm{~g} / \mathrm{s}\left(\sim 0.315\left|\Psi_{\min }\right|\right)$, respectively. Interestingly, the oscillation amplitude of the interface speed is less than $10^{-4} \mu \mathrm{m} / \mathrm{s}$, which is much smaller than the pulling speed $(2 \mu \mathrm{m} / \mathrm{s})$ for both cases. This indicates that the angular vibration should not cause significant growth striations even at low frequency (say $5 \mathrm{~Hz}$ ).

We believe that the discrepancy between the results from DNS and SBLA was mainly from the finite thickness of the viscous boundary layer and the non-trivial amplitude; $\delta \sim 0.4$ and $0.2 \mathrm{~mm}$ for 5 and $20 \mathrm{~Hz}$, respectively. Decreasing the vibration amplitude or increasing frequency, the discrepancy decreases. Although an accurate simulation has not been achieved, for the present study, we can conclude that SBLA was a reasonable approximation for simulation, at least for $20 \mathrm{~Hz}$, where the simulated results are consistent with the experimental observations. For $5 \mathrm{~Hz}$, the simulation result was not satisfactory. More importantly, the calculation using SBLA, over predicted the flow intensity. Nevertheless, SBLA remains a feasible approach to the present simulation. We also carried out simulation for $10 \mathrm{~Hz}$ for the growth condition, and the interface still remained planar.

The superheating gradients $\Delta G(\Delta G=\mathrm{d} T / \mathrm{d} z-m \mathrm{~d} C /$ $\mathrm{d} z$ ) at the center of the interface for different cases were also calculated, as shown in Fig. 6; $m$ is the slope of the liquidus line obtained from the equilibrium phase diagram. Constitutional supercooling occurs when $\Delta G$ becomes negative. The curves for 0 and $5 \mathrm{~Hz}$ DNS are quite similar. Once the melt was supercooled for a certain degree, the simulation broke down easily; in reality, the morphological instability occured when the supercooling overcame the interfacial energy. On the contrary, at high frequencies or $5 \mathrm{~Hz}$ with SBLA, no supercooling existed in front of the interface. Again, SBLA at $5 \mathrm{~Hz}$ over estimated the effect of angular vibration. For 10 and $20 \mathrm{~Hz}$, the simulations based on SBLA are consistent with the experimental observations.

The calculated radial acetone segregations $\left(\Delta C_{\mathrm{c}} / C_{0}\right)$ are shown in Fig. 7; $\Delta C_{\mathrm{c}} / C_{0} \equiv K\left(\left.C^{*}\right|_{r^{*}=1}-\left.C^{*}\right|_{r^{*}=0}\right)$. Severe radial solute segregations were found for 0 and $5 \mathrm{~Hz}$ (DNS). On the other hand, with enough angular vibration, the radial segregation profiles were about the same having higher acetone concentration near the ampoule wall. As a result, the acetone accumulation at the interface center was removed. The spreading out of the acetone reduced the local acetone concentration and thus significantly improved the morphological stability. Also, as shown in the figure, the acetone accumulation was reduced by $5-\mathrm{Hz}$ angular vibration based on DNS simulation. The simulation result at higher frequencies should approach to the results of SBLA. This further supports the validity of SBLA for high frequencies in the present case.

In addition to the reduction of radial segregation, improvement in axial uniformity is necessary for the

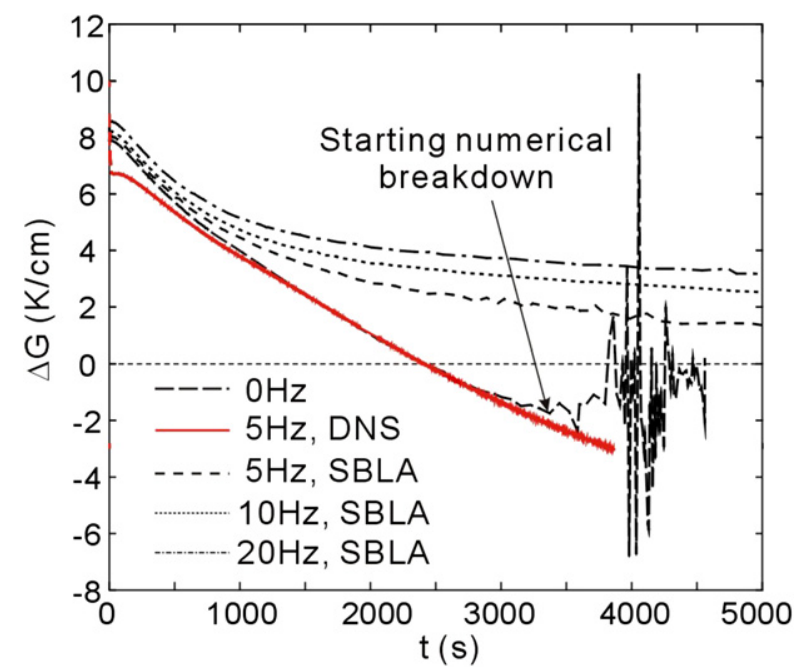

Fig. 6. Calculated superheating gradients at the center of the growing interface for various conditions; the wiggle for $5 \mathrm{~Hz}$ (DNS) is due to numerical breakdown. 


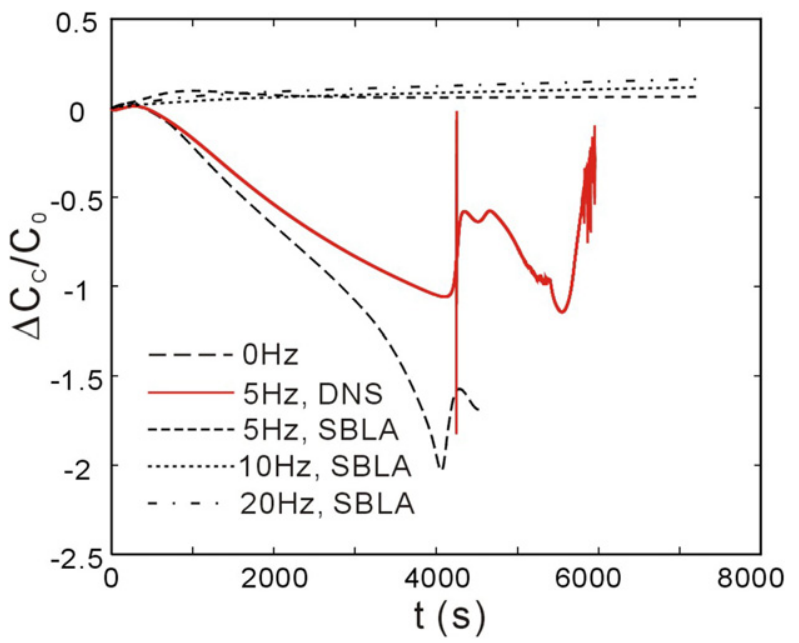

Fig. 7. Calculated radial segregations $\left(\Delta C_{\mathrm{c}} / C_{0}\right)$ at various conditions; numerical breakdown is clear for 0 and $5 \mathrm{~Hz}$ DNS after $t=4000 \mathrm{~s}$; $\Delta C_{\mathrm{c}} / C_{0}=K\left(\left.C^{*}\right|_{r^{*}=1}-\left.C^{*}\right|_{r^{*}=0}\right)$.

Bridgman crystal growth. As mentioned previously, reducing solute global mixing is a simple way to improve the uniformity if the growth distance is much longer than the diffusive distance. Fig. 8 shows the calculated axial solute segregations (in radial average) in the growing crystal. Interestingly, for $5 \mathrm{~Hz}$, the segregation behaviors of DNS and SBLA are quite similar; both are more diffusive (less convective transport) than the others. On the other hand, the segregation profiles for 0,10 , and $20 \mathrm{~Hz}$ are all similar having a better solute mixing. It is thus clear that the angular vibration modified the flow structure and tended to enhance solute mixing near the growing interface. Therefore, in order to keep a more diffusive growth condition with a less global solute mixing, a high frequency and low amplitude vibration is recommended for reducing the axial segregation.

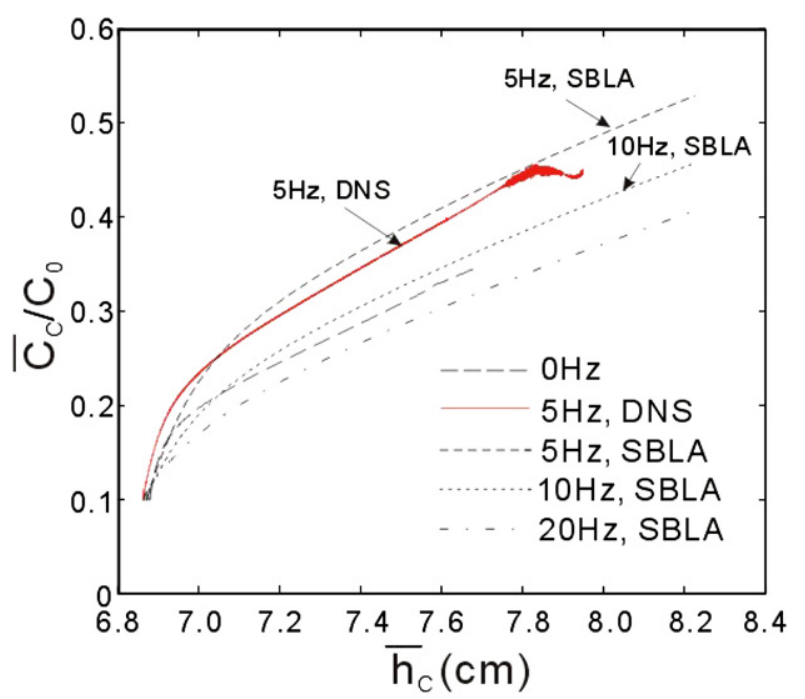

Fig. 8. Calculated axial acetone segregation (normalized radial averaged concentration) for various vibration conditions.

\section{Conclusion}

The effect of angular vibration on the flow, morphology, and solute segregation during vertical Bridgman crystal growth of SCN containing $0.02 \mathrm{wt} . \%$ acetone was investigated. Both direct numerical simulation (DNS) and Schlichting boundary layer approximation (SBLA) were considered in the numerical modeling and compared with the experimental observations. The simulated results are consistent reasonably well with the experimental results. However, as anticipated, SBLA was only suitable for high frequencies with low amplitudes. At a low frequency, e.g., $5 \mathrm{~Hz}$, SBLA was found erroneous. Nevertheless, based on the simulated results and the transparent experiments, the angular vibration technique was proven to be effective in the flow and segregation control for the vertical Bridgman crystal growth.

\section{Acknowledgement}

This work was supported by the National Science Council of the Republic of China and the Russian Foundation for Basic Research.

\section{References}

[1] J.C. Brice, Crystal Growth Processes, Jone Wiley \& Sons, New York, 1986.

[2] E. Monberg, Bridgman and related growth techniques, in: D.T.J. Hurle (Ed.), Handbook of Crystal Growth 2a: Basic Techniques, North-Holland, Amsterdam, 1994, pp. 51-97.

[3] W.A. Tiller, K.A. Jackson, J.W. Rutter, B. Chalmers, The redistribution of solute atoms during the solidification of metals, Acta Metall. 1 (1953) 428-437.

[4] W.W. Mullins, R.F. Sekerka, Stability of a planar interface during solidification of a dilute binary alloy, J. Appl. Phys. 35 (1964) 444451.

[5] N.B. Singh, S.S. Mani, J.D. Adam, S.R. Coriell, M.E. Glicksman, W.M.B. Duval, G.J. Santaro, R. DeWitt, Direct observations of interface instability, J. Cryst. Growth 166 (1996) 364-369.

[6] C.W. Lan, C.Y. Tu, Morphological instability due to double diffusive convection in directional solidification: the pit formation, J. Cryst. Growth 220 (2001) 619-630.

[7] P. Dold, K.W. Benz, Rotating magnetic fields: fluid flow and crystal growth applications, Prog. Cryst. Growth Charact. Mater. (1999) 7-38.

[8] D.T.J. Hurle, R.W. Series, Use of a magnetic field in melt growth, in: D.T.J. Hurle (Ed.), Handbook of Crystal Growth 2a, North-Holland, Amsterdam, 1994, pp. 259-286.

[9] H. Rodot, L.L. Regel, G.V. Sarafanov, H. Hamidi, I.V. Videskii, A.M. Turtchaninov, Cristaux de plomb élaborés en centrifugeuse, J. Cryst. Growth 79 (1986) 77-83.

[10] J. Friedrich, J. Baumgartl, H.-J. Leister, G. Mueller, Experimental and theoretical analysis of convection and segregation in vertical Bridgman growth under high gravity on a centrifuge, J. Cryst. Growth 167 (45) (1996) 45-63.

[11] C.W. Lan, Y.W. Yang, H.Z. Chen, I.F. Lee, Segregation and morphological instability due to double-diffusive convection in rotational directional solidification, Metall. Mater. Trans. A 33 (2002) 3011-3017.

[12] H.J. Scheel, Flux growth of large crystals by accelerated cruciblerotation technique, J. Cryst. Growth 13 (1971) 304-306. 
[13] W.C. Yu, Z.B. Chen, W.T. Hue, B. Roux, T.P. Lyubimova, C.W. Lan, Reversing radial segregation and suppressing morphological instability during Bridgman crystal growth by angular vibration, J. Cryst. Growth 271 (2004) 474-480.

[14] C.W. Lan, Flow and segregation control by accelerated rotation for vertical Bridgman growth of cadmium zinc telluride: ACRT versus vibration, J. Cryst. Growth 274 (2005) 379-386.
[15] C.W. Lan, M.C. Liang, Modeling of dopant segregation in vertical zone-melting crystal growth, J. Cryst. Growth 186 (1998) 203-213.

[16] W.L. Nyborg, Acoustic streaming, in: W.P. Wason (Ed.), Physical Acoustics, Academic Press, 1965.

[17] Y.W. Lee, R. Anauth, W.N. Gill, Preparation of ultra-pure succinonitrile by countercurrent distillation for crystal growth, Chem. Eng. Commun. 152 (1996) 41-52. 